\title{
PERSPECTIVASY PROPUESTAS COMUNITARIAS EN LA VALORACIÓN DEL PATRIMONIO ARQUEOLÓGICO. UN CASO EN LA CIUDAD DE CASMA, COSTA NOR-CENTRAL DE PERÚ
}

\author{
JoRge GAMBOA VELÁZOUEZ \\ UniVERSIDAD NACIONAL SANTIAGo ANTÚNEZ DE MAYOlO \\ jgamboavelasquez@yahoo.com
}

\section{RESUMEN}

Las últimas décadas han visto tanto la revisión crítica y ampliación de los fundamentos teóricos de la arqueología como un amplio debate sobre el involucramiento de diversos grupos y agendas sociales en la práctica de esa disciplina. Esta contribución explora los resultados de la interacción entre la investigación de las sociedades prehispánicas y las iniciativas locales orientadas a la promoción del patrimonio arqueológico en la costa de Ancash, Perú. Para ello se analiza el acercamiento realizado a partir del año 2011 por diversos actores sociales dedicados al estudio, preservación y difusión del patrimonio arqueológico de esa parte de la costa Pacífica de Sudamérica. El caso presentado también permite examinar el aporte de la arqueología a la (re)construcción de una historia prehispánica regional poco representada en los discursos oficiales de la educación peruana.

Palabras clave: Perú, costa de Ancash, arqueología, patrimonio cultural, educación, público.

\section{Abstract}

In the last few decades archaeological perspectives have expanded to incorporate the involvement of different social groups and agendas. This contribution explores the results of the interaction between archaeological research and contemporary local initiatives that promote archaeological heritage in the coastal region of Ancash. To fulfill this objective I analyze the role of different stakeholders in the management of archaeological heritage in that part of northern Peru. The case study permits an assessment of how archaeology can contribute to the (re)construction of a regional Pre-Columbian history that is underrepresented in official discourses of Peruvian public education.

KeYword: Peru, Ancash coast, archaeology, cultural heritage, education, public. 
La presentación pública del avance de las investigaciones arqueológicas es un hecho cada vez más frecuente en el norte peruano. Se puede citar como ejemplos de esta práctica las conferencias, talleres y exposiciones realizadas de manera corporativa o aislada en las regiones Lambayeque, La Libertad y Ancash por proyectos de investigación y entidades estales (en el último caso por universidades, el Ministerio de Cultura y las Unidades Ejecutoras). Por otro lado, también es evidente que los resultados de esas reuniones no siempre llegan a ser publicados o a ser presentados en un sitio web, alcanzando, en consecuencia, un impacto básicamente local y pasajero. A pesar de esa limitación, estos encuentros se han convertido en espacios para la interacción entre los diversos actores sociales involucrados en la investigación, preservación y difusión del patrimonio arqueológico y cultural. El caso examinado en esta contribución corresponde a un simposio regional realizado algunos años atrás en la ciudad de Casma, un encuentro en el que diversos esfuerzos confluyeron para alcanzar las metas del encuentro y dialogo entre público e investigadores.

\section{Los Organizadores}

El 2 de julio del 2011 se desarrollo en la ciudad de Casma el "Primer Ciclo de Conferencias sobre la Arqueología de la Costa de Ancash", un evento organizado por iniciativa de la Casa de la Cultura de Casma, una entusiasta organización civil local, con el apoyo del Museo Regional Max Uhle-Cerro Sechín del Ministerio de Cultura de Perú1. La jornada académica y de difusión permitió a diversos arqueólogos peruanos y extranjeros compartir sus datos y experiencias sobre la evolución de la complejidad social prehispánica en la vertiente del Océano Pacifico de Ancash, zona conformada por los valles de Santa, Lacramarca, Nepeña, Casma, Culebras, Huarmey y Fortaleza y que se extiende entre el litoral y los $2500 \mathrm{msnm}$ (Fig. 1). El encuentro también fue concebido como un reconocimiento a la trayectoria de la destacada arqueóloga casmeña Dra. Rosa Fung Pineda. La jornada cultural incluyó la presentación de doce ponencias, la mayoría correspondiendo a proyectos arqueológicos en actividad. El evento recibió el apoyo económico y logístico de la Municipalidad Provincial de Casma y diversas entidades privadas de Casma -restaurantes y operadores turísticos locales y una empresa multinacional a cargo de la renovación de carreteras-, demostrando la capacidad de convocatoria que este tipo de actividades puede llegar a alcanzar.

\section{El Contexto Social}

El público que acudió a la reunión estuvo formado por pobladores de Casma con interés en historia y turismo, estudiantes y profesores de escuelas primarias y secundarias, funcionarios civiles y los propios arqueólogos (Fig. 2). Vale la pena recordar que, al igual que en el resto de Perú, los libros de historia empleados en las escuelas de la costa de Ancash hacen escasas referencias a los sitios y sociedades prehispánicas locales. La casi completa ausencia de datos sobre las evidencias arqueológicas (prehispánicas y coloniales) locales ocurre a la par de una aproximación esporádica, o poco reflexiva,

1. Los antecedentes directos de las políticas culturales peruanas en torno a la preservación y valoración del patrimonio arqueológico se encuentran en el establecimiento en 1929 del Patronato de Cultura (Ley 6634), un organismo que desde sus sedes en Lima, Cusco y otras ciudades peruanas dirigió las primeras labores organizadas de protección de los sitios prehispánicos nacionales. La Casa de la Cultura del Perú y sus sedes regionales fueron formalizadas en la década de 1960 sobre un modelo parcialmente inspirado en el entonces naciente Ministerio de Cultura de Francia. En 1971 el Patronato de Cultura dio paso al Instituto Nacional de Cultura del Perú (actual Ministerio de Cultura del Perú). La Casa de la Cultura de Casma es una agrupación civil cuyo origen no tiene vínculos directos con las entidades anteriormente mencionadas. Un resumen de las actividades realizadas por esa 


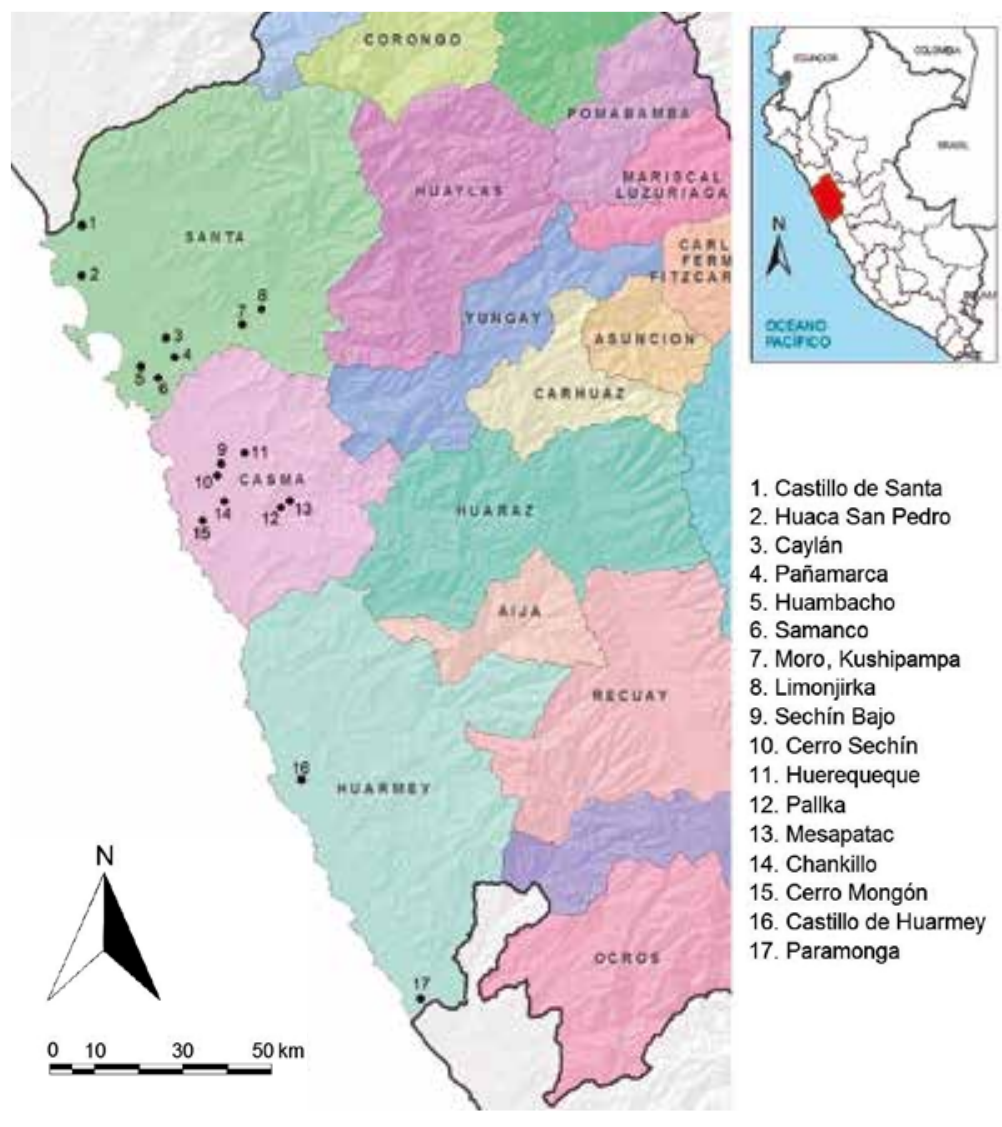

Figura 1. Mapa de la costa de Ancash con ubicación de sitios mencionados en el texto. Elaboración por J. Gamboa.

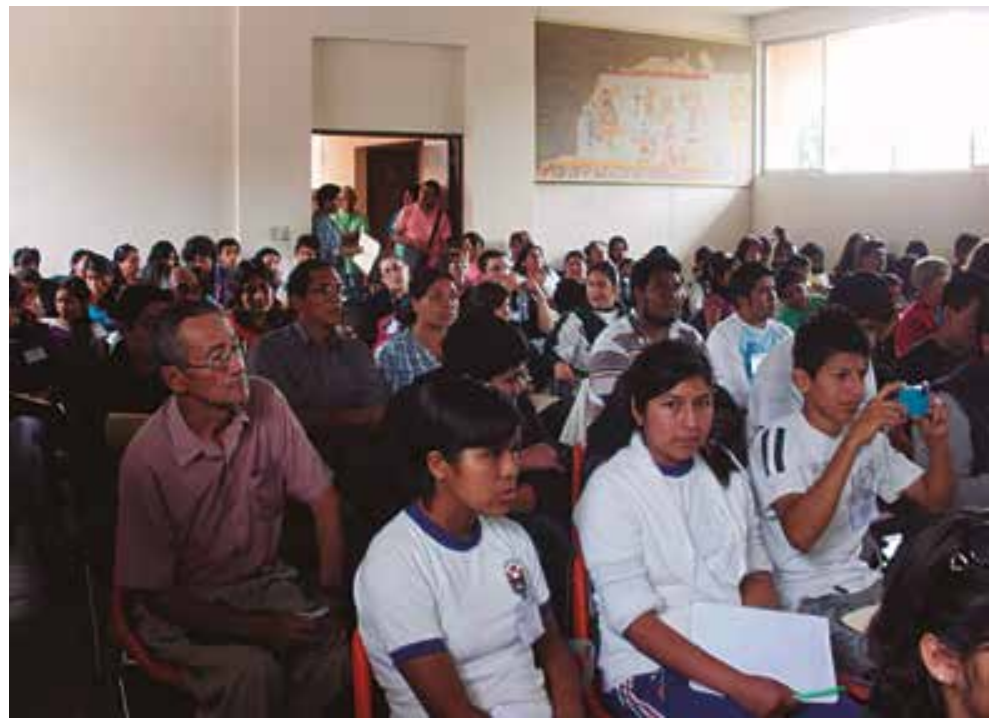

Figura 2. Público reunido en el simposio de Casma de julio del 2011. Fotografía por J. Gamboa 
al estado y valor del patrimonio arqueológico promovida por las políticas educativas y los agentes educativos estatales y privados (Gamboa 2015:4, 65, 76; Vogel y Pacifico 2004:49). Hasta fines del año 2015 en ninguna de las provincias de la costa de Ancash se habían implementado proyectos de arqueología comunitaria. El número de experiencias de participación comunitaria en la conducción de museos y proyectos de investigación es hasta el presente también limitado ${ }^{2}$. En Casma esas carencias son solucionadas en parte por las visitas escolares y turísticas al sitio Cerro Sechín y su museo de sitio. En otras partes del área no existen esas posibilidades de acercamiento al patrimonio arqueológico. En realidad, para muchos de los asistentes al evento, esta fue una primera oportunidad para apreciar las rotundas diferencias entre el conocimiento adquirido a través de la investigación arqueológica y lo que, con suerte, es incluido en la enseñanza pública de la historia.

En la cotidianeidad de las escuelas de los poblados rurales y periferias urbanas de Perú la norma es hallar recursos bibliográficos limitados y alejados de la realidad local. Los manuales educativos empleados muestran (de forma breve) solo los principales sitios arqueológicos nacionales. En contraste, esas mismas áreas suelen colindar con asentamientos prehispánicos y aún con edificios y rasgos de los periodos Colonial y Republicano, sitios que con frecuencia se encuentran sometidos a daños por saqueadores y una presión territorial cada vez mayor provocada por la expansión de zonas agrícolas y urbanas (Fig. 3). En el valle de Casma la depredación de sitios arqueológicos prosigue, habiéndose sumado al saqueo de cementerios prehispánicos el desmontaje de estructuras de adobe y piedra y el riego (mediante canales o tecnificado) sobre espacios con evidencias prehispánicas, una situación que también ocurre en los valles vecinos. ¿Qué provoca tal avance de la destrucción del patrimonio arqueológico? En la costa de Ancash las causas son múltiples, pero suelen girar alrededor de la extracción y comercio ilegal de artefactos prehispánicos y la rápida transformación de paisajes culturales y sitios arqueológicos. Ese último factor está a su vez relacionado a una mayor disponibilidad dentro de la región de capitales económicos producido por la agricultura de exportación y la minería (legal, ilegal e informal). Las deficiencias ya mencionadas del sistema educativo y el escaso apoyo del estado a las instituciones culturales regionales son sin duda otros factores a tomar en cuenta.

Las iniciativas económicas basadas en el turismo forman parte de las agendas locales sobre el patrimonio arqueológico, aunque, como veremos, su relación con la difusión y manejo de sitios patrimoniales varía en cada región. En la costa de Ancash la industria turística a cargo de operadores locales está mediana o escasamente desarrollada y se encuentra hasta ahora enfocada en paisajes naturales presentados a un público mayormente nacional (tanto de la región como procedente de ciudades como Lima y Trujillo). El bajo número de empresas locales dedicadas al turismo ha visto en años recientes un incremento gradual, especialmente en Chimbote, Casma y, más recientemente, Huarmey.

2. Ver Onuki (2006) para el museo de sitio de Kuntur Wasi y Montenegro y Rivolta (2013) para un caso de museo comunitario en la sierra noroccidental de Argentina. En la costa de Ancash existen varios centros educativos que albergan colecciones arqueológicas formadas por materiales carentes de contextualización y procedentes de contextos depredados. Dos ejemplos son las colecciones arqueológicas de las escuelas públicas de Vinzos, en la Provincia de Santa (http://santa-ancash-peru.blogspot.com/2009/09/vinzos-ancashtodo-acerca-de.html, accedido: 25 de abril del 2016) y de Nivín, en Casma (https://www.youtube.com/watch?v=uZo5c8zFnBg y https:// piaraperublog.wordpress.com/2015/07/24/una-sorpresa-arqueologica-en-nivin-peruan-archaeological-surprise-in-nivin-peru/\#english, accedidos: 25 abril 2016). En el 2015, la iglesia, donantes internacionales y la Municipalidad de Moro, en el valle de Nepeña, apoyaron la formación en esa ciudad del "Museo de las Tecnologías Andinas", inaugurado ese mismo año y organizado sobre la base de una colección arqueológica local (A. Herrera, com. pers. 2015). Originados en esfuerzos aislados inicialmente desvinculados de la investigación arqueológica, 

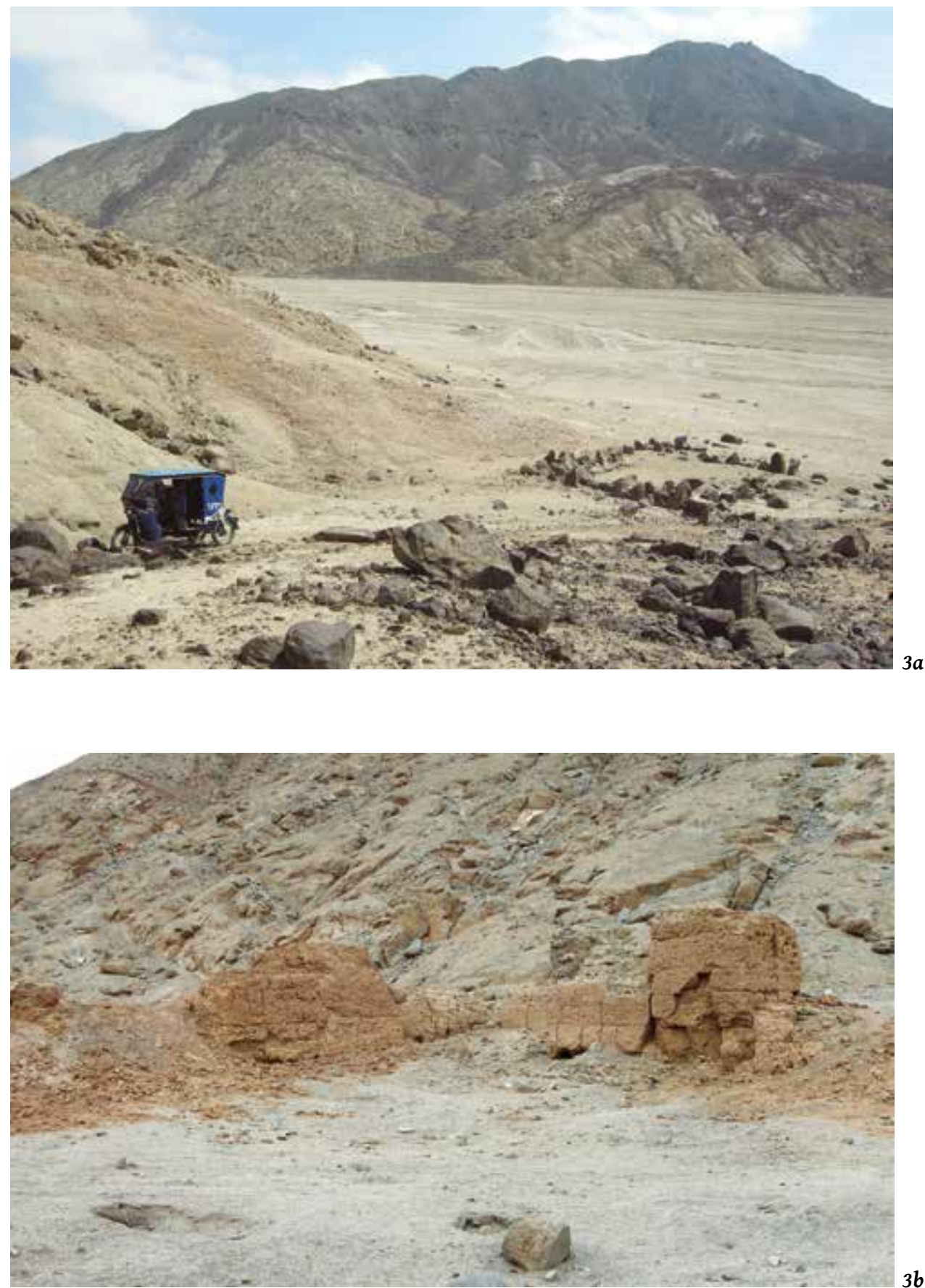

Figura 3. Estructuras arqueológicas en proceso de saqueo y destrucción en el valle de Casma. a) Recinto de piedras en sector Lomo de Camello, b) edificio de tapial en sitio al noroeste del sector La Cantina y Chankillo. Fotografías por J. Gamboa, 2009 y 2012. 
Parte de ese cambio ha estado relacionado a la ampliación de la oferta turística en torno al patrimonio arqueológico regional. En la costa de Ancash solo los sitios Cerro Sechín, Pañamarca y Paramonga, todos asentamientos prehispánicos con carácter monumental, tienen la condición de lugares abiertos al público bajo la protección del Ministerio de Cultura del Perú. El número de visitantes a Cerro Sechín -el único de la costa de Ancash que recibe visitantes de manera regular- fue de 23,000 en el año 2015, cantidad aún lejana a los 72,000 turistas que arribaron a Chavín de Huántar, en la parte altoandina de Ancash, durante la misma temporada. En contraste a esos centros monumentales, la mayoría de sitios prehispánicos del área carecen de control permanente y se encuentran afectados por los factores negativos antes señalados. El estado de conservación y valoración del patrimonio arqueológico en la costa de Ancash revela no solo el impacto aún menor de los espacios museográficos y zonas arqueológicas en la sociedad regional sino también un desarrollo limitado de las industrias turísticas locales. Estas características son aún más marcadas cuando el panorama actual del manejo de recursos culturales en la costa de Ancash es comparado con aquel conducido en otras partes de la costa norte y nor-central peruana (Gamboa 2015:59-67; Wester 2010:217-237).

En el plano local, la situación descrita no debe ser entendida como prueba de una falta de relación entre pobladores y sitios arqueológicos. Los estudios de campo demuestran lo contrario. Por ejemplo, la investigación de Pacífico (2008) sobre la interacción entre comunidades rurales modernas y sitios prehispánicos en el sector Purgatorio del valle de Casma demostró que los espacios arqueológicos son apreciados paralelamente como símbolos de ancestralidad y otredad y como referentes materiales de un pasado ubicuo pero a la vez distante. Ese mismo estudio evidenció que los sitios arqueológicos no son concebidos de manera uniforme, existiendo diferentes perspectivas locales -individuales o comunitarias- sobre el potencial del patrimonio arqueológico en: i) el mejoramiento de las condiciones de educación, ii) la identidad distrital, y iii) la ampliación del espectro de actividades económicas zonales (algo que comprende diversas posiciones sobre las posibilidades de un desarrollo turístico). Más allá de ello, y al igual que en el resto del globo, los sitios arqueológicos, grandes y pequeños, suelen ser escenario de memorias y microhistorias, llegando a convertirse en componentes centrales de las narrativas y diálogos locales sobre el pasado y presente.

\section{El Evento}

La mayoría de presentaciones en el simposio en Casma estuvieron enfocadas en sitios y áreas de ocupación de los periodos Arcaico (10000-1600 aC) y Formativo (1600-100 aC) ancashino, demostrando la importancia académica dada a la investigación del poblamiento temprano y la emergencia de sociedades complejas en esa parte del territorio Andino. En efecto, en las últimas décadas el estudio de las sociedades tempranas de los valles costeños de Santa, Nepeña y Casma ha aportado numerosos datos sobre los procesos de cambio cultural transcurridos entre las poblaciones cazadoras-recolectoras del área y la fundación de los primeros centros ceremoniales regionales. Por otro lado, esos trabajos han pasado a conformar una valiosa y necesaria contraparte de información y discusión a las investigaciones arqueológicas conducidas en Chavín de Huántar y el resto de la sierra ancashina (Bria 2013; Lau 2010; Rick et al. 2009).

En el encuentro de Casma se presentaron ponencias dedicadas a los sitios Sechín Bajo, Huerequeque y Chankillo en Casma; Caylán y Kushipampa en Nepeña; y El Castillo de Santa en Santa. Asimismo, se expusieron los resultados de prospecciones realizadas en Yaután en la parte alta del valle de Casma y en la cuenca alta de Nepeña. En conjunto, las ponencias presentadas expusieron los avances logrados en la reconstrucción de una historia regional extendida entre el décimo milenio a.C. y 1000 d.C. y cubrieron diversos aspectos de la organización sociopolítica, ideología y fundamentos económicos 
de las poblaciones prehispánicas de las cuencas de Santa, Nepeña y Casma. Otro tema discutido fue el rol del Museo de Cerro Sechín en el desarrollo de la arqueología regional y en la integración entre la investigación del pasado y la sociedad moderna.

\section{(Re)Construyendo una Historia Regional}

La arqueología es una fuente privilegiada de información sobre el pasado de la humanidad. A pesar de la separación en la historiografía tradicional entre "prehistoria" -entendida como el periodo de tiempo correspondiendo a sociedades sin escritura- e "historia", la mayoría de investigadores están actualmente de acuerdo en que esa separación corresponde solo a la naturaleza de las fuentes de datos (Mamani 1996; Neves 1998). Los estudios arqueológicos permiten la reconstrucción e interpretación del transcurrir de las sociedades humanas a través del tiempo y el espacio. La investigación de las formaciones sociales prehispánicas implica en ese sentido la definición del surgimiento y transformación de esferas de interacción, estilos locales e identidades sociales. A través de estudios de campo y gabinete ha sido posible comprender realidades sociales locales y sistemas regionales de intercambio económico e interacción política. Al énfasis en la arqueología Andina en los procesos sociales y los cambios en diseño y función experimentados por los asentamientos se han sumado diversas aproximaciones a los rasgos biográficos e identitarios de personajes específicos (p. ej., Alva y Donnan 1993; Marcus 2015; Nelson et al. 2000). En todas esas variantes, la arqueología ha contribuido activamente al reconocimiento de la materialidad y simbolismo pasado como parte inherente de las realidades sociales, económicas e ideológicas presentes. La visión del pasado regional a través de la arqueología ha incluido así la comprensión de las formaciones sociales anteriores al siglo XVI, con esta disciplina buscando superar la división metodológica entre fuentes arqueológicas, documentales y etnológicas en la reconstrucción de la historia regional. El simposio de Casma del 2011 fue un ejemplo de esa posibilidad.

La primera ponencia presentó un panorama detallado sobre la ocupación humana en el área de Casma entre fines del Pleistoceno, tras la última glaciación, e inicios del Holoceno, la era geológica actual. El Precerámico en el valle bajo de Casma fue tratado por Santiago Uceda, quien describió las manifestaciones Paijanense (circa 9000-6000 a.C.), Mongoncillo (6000-3500 a.C.) y del Arcaico Tardío (3500-1600 a.C.) registradas en 1982 y 1983 entre Playa Tortugas y Las Aldas. El análisis de la distribución de sitios y utillajes líticos permitió a Uceda (1992) proponer la existencia de especialización económica por parte de las poblaciones sucesivamente implicadas en la explotación de los recursos del litoral y lomas costeras del valle. Respecto a la ocupación Paijanense, Uceda comparó los artefactos líticos de esa tradición en Casma y Chicama, definiendo similitudes y diferencias tanto en la disponibilidad de materias primas como en el rango de actividades desarrolladas. Uceda también señaló la existencia en la margen sur del valle bajo de Casma de una sociedad agrícola del Arcaico Tardío caracterizada por la producción de herramientas de piedra percutida usadas para remover la tierra y extraer los primeros productos agrícolas cultivados en la zona; otro resultado fue la identificación de maíz entre los cultígenos de ese periodo. A través de estos últimos datos, los aportes de Uceda sirvieron de introducción a la siguiente presentación, la cual examinó el surgimiento de los centros ceremoniales en la región nor-central Andina.

Peter Fuchs, Jesús Briceño y Germán Yenque expusieron la secuencia constructiva de Sechín Bajo, el sitio actualmente considerado poseedor de algunas de las evidencias más tempranas de arquitectura ceremonial y pública en Sudamérica y a nivel global. Los miembros del Proyecto Sechín Bajo expusieron sus datos sobre la evolución del sitio, el cual hacia 4000 a.C. empezó a presentar espacios rituales en forma de patios circulares hundidos (Fuchs et al. 2010). Construidas por las primeras co- 


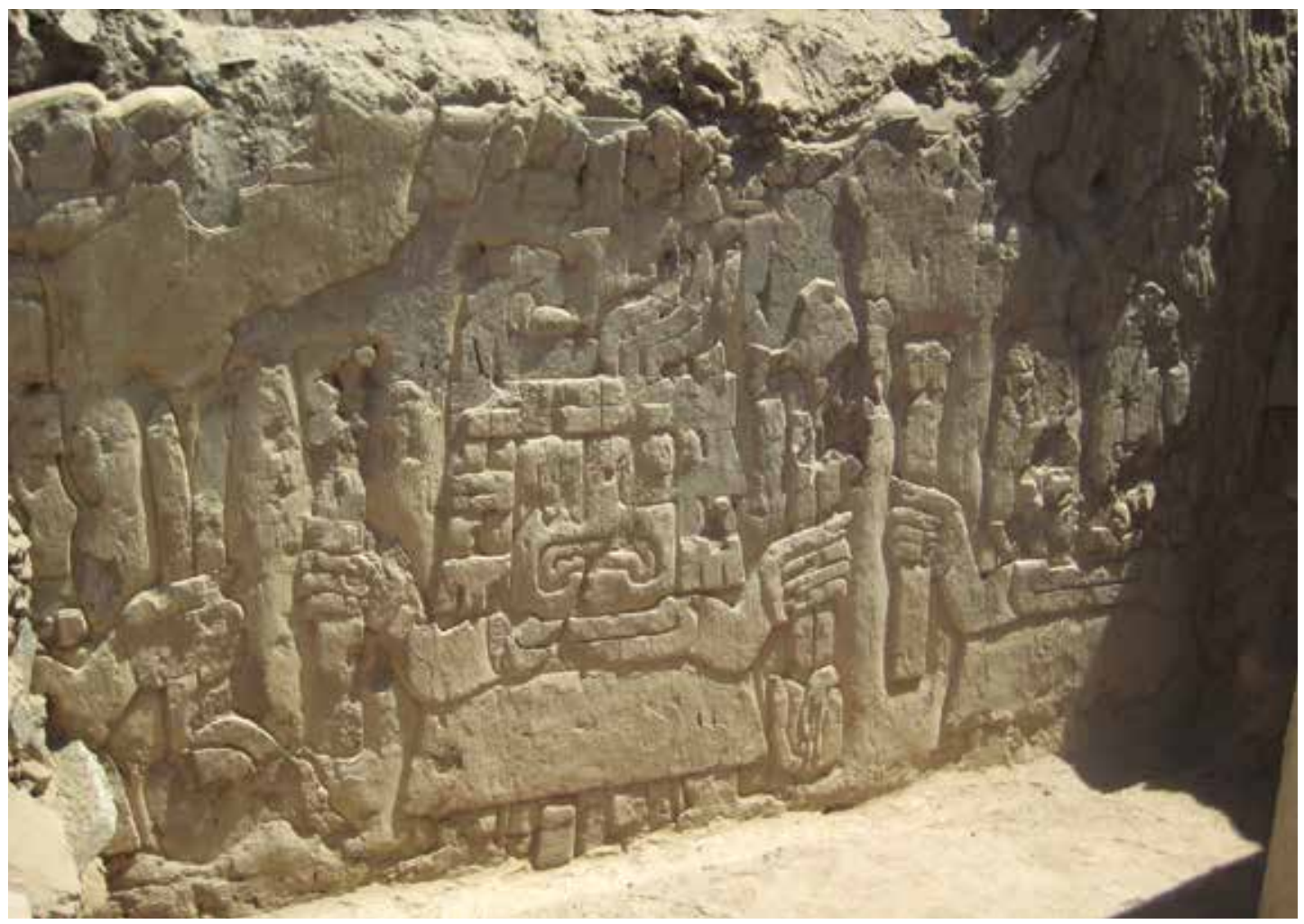

Figura 4. Relieves de barro de Sechín Bajo, Tercer Edificio (1500-1200 aC). Fotografía cortesía de Peter Fuchs, Proyecto Arqueológico Sechín Bajo.

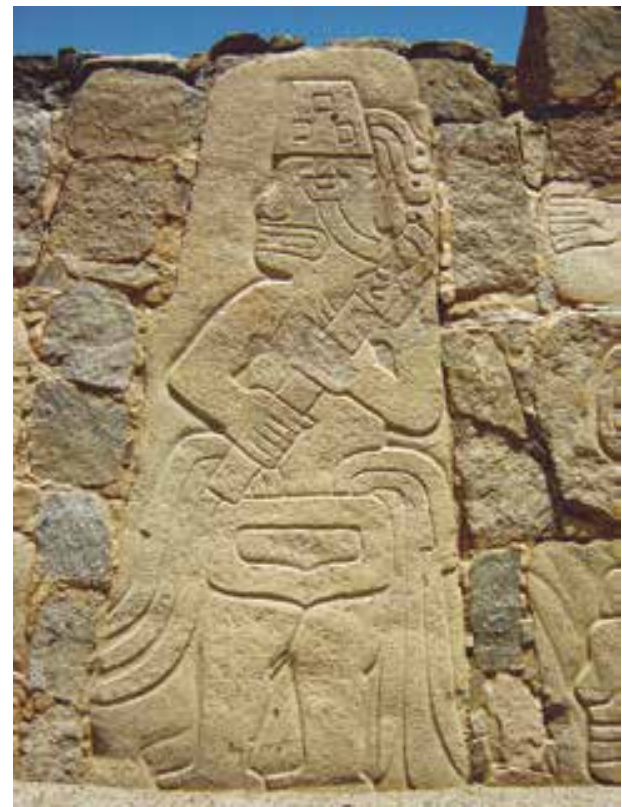

Figura 5.- Esculturas líticas de Mesapatac (izq.) y Cerro Sechín (der.). Fotografías por J. Gamboa, 2012. 
munidades aldeanas con economía agrícola del área, esos patios eran periódicamente renovados y reconstruidos, con cada episodio mostrando innovaciones de diseño constructivo y empleo de materiales. Posteriormente, esas áreas ceremoniales fueron recubiertos por un conjunto arquitectónico con salas de esquinas curvas y muros elevados provistos de nichos, una estructura cuya construcción y uso ha sido datada entre 1600 y 1500 a.C. y que data del periodo de introducción de la cerámica en la costa peruana. Alrededor de 1500 a.C. un tercer edificio fue erigido en proximidad a la construcción anterior. Ese último conjunto devendría, a través de su secuencia de crecimiento arquitectónico, en una de las plataformas de mayores dimensiones del periodo Formativo (1600-100 aC) del valle de Casma y la costa peruana (Fig. 4).

El periodo Formativo comprendió el tiempo de consolidación y transformación final de los sistemas sociopolíticos establecidos en el Arcaico Tardío. Ambos periodos deben ser vistos por lo tanto como estrechamente relacionados. La transición entre ambos periodos en los sitios Mesapatac y Pallka del distrito de Yaután en la sección media-alta del río Casma, fue evaluada por Mónica Suárez. En el año 2003 Mesapatac fue escenario del hallazgo por agricultores locales de tres relieves líticos vinculados estilísticamente a la tradición iconográfica de Cerro Sechín en el valle bajo de Casma (Fig. 5). Los monolitos fueron posteriormente publicados por Suárez, quien identificó las imágenes de un felino sobrenatural con apéndices escalonados en el lomo y una cabeza humana al interior del cuerpo, una serpiente bicéfala con apéndices bucales, y la figura naturalista de un felino. Esas litoesculturas habrían procedido de un edificio contemporáneo, por analogía estilística, con Cerro Sechín y perteneciente al tiempo entre 2000 y 1500 a.C.

Suárez también presento información sobre Pallka, un importante complejo arquitectónico del Formativo Medio y Tardío del área de Yaután reconocido inicialmente por Julio C. Tello (1956). Uno de los asentamientos formativos mejor conservados de la costa nor-central, Pallka está formado por una plataforma con escalinatas centrales, plaza rectangular delantera, una plaza circular hundida lateral, terrazas y caminos. Reseñando sus trabajos en el sitio durante los años 2000 y 2001, Suárez describió las formas cerámicas presentes en Pallka y señaló las relaciones estilísticas de esos materiales con las tradiciones alfareras del sitio altoandino de Chavín de Huántar y los asentamientos formativos del valle bajo de Casma (Chávez 2011; Suárez 2008) (Fig. 6).

La investigación en el sitio Huerequeque fue presentada por Thomas y Shelia Pozorski, quienes expusieron los resultados de sus primeras campañas de excavación en ese complejo arquitectónico Formativo del valle medio del río Sechín, el ramal norte de la cuenca del valle de Casma (Pozorski y Pozorski 1987:82-85). La parte central de Huerequeque está conformada por un conjunto monumental del Formativo Medio provisto de una plataforma, un patio rectangular hundido y una plaza circular. Los nuevos datos para el asentamiento indican que la plataforma habría estado en proceso de renovación al momento de ser abandonada. La plaza rectangular evidenció sellar una plaza circular más temprana cuidadosamente rellenada con piedras de grandes dimensiones. La secuencia constructiva registrada indicó una sucesión de eventos arquitectónicos asociados sucesivamente a la construcción de una primera plaza circular, un edificio aterrazado, y las dos plazas tardías, con una fase final de recubrimiento parcial del conjunto y posterior abandono.

Los trabajos dedicados a la parte final del periodo Formativo de los valles de Casma y Nepeña fueron presentados a continuación. David Chicoine describió las características del desarrollo de Caylán, un asentamiento con características urbanas localizado en el valle bajo de Nepeña. Caylán se constituyo en el principal centro residencial y administrativo del área entre los siglos VI a II a.C., presentando numerosos conjuntos habitacionales y ceremoniales con patios interiores, pilares y muros decorados con frisos geométricos (Chicoine e Ikehara 2010). La organización de esos espacios arquitectónicos 
señala el desarrollo de ceremonias de interacción y competencia comunitaria así como la jerarquización de los linajes participantes, los cuales habrían estado integrados en un complejo paisaje edificado y simbólico materializado en una extensa red urbana de barrios, calles y templos (Fig. 7). Esta entidad sociopolítica pudo encabezar a una serie de comunidades cercanas más pequeñas, como las de Huambacho, Pañamarca y Samanco. Con posterioridad al simposio de Casma, la investigación en Caylán permitió constatar el uso de camélidos sudamericanos (Lama glama y Vicugna pacos) en esa antigua capital del valle de Nepeña y demostró la importancia de esas especies en la economía política e ideología religiosa de ese asentamiento y el sitio Huambacho (Szpat et al. 2015).

Hugo Ikehara expusó sobre la ocupación del Formativo Tardío y Final (600-100 a.C.) en Moro, valle medio de Nepeña, presentando los resultados de sus excavaciones en Kushipampa, un asentamiento donde se encuentra un conjunto de edificios con portadas monumentales de piedra finamente tallada así como diversas áreas de vivienda y descarte de desechos (Ikehara 2010). Asimismo, se presentaron datos sobre San Isidro, otro de los sitios tempranos del área, señalándose para ese lugar la presencia de cerámica y arquitectura Formativa y su posible rol como santuario en un territorio políticamente fragmentado. A nivel sub-regional, Kushipampa habría dominado la zona alrededor de la actual ciudad de Moro, interactuando con los grupos del valle bajo de Nepeña y las sociedades serranas de la cercana Cordillera Negra. Los datos de Chicoine e Ikehara pusieron en relieve un tiempo y un espacio marcados por la diversidad de formas de organización comunal pero también por el incremento del militarismo y agresiones interzonales que caracterizaron a la última parte del periodo Formativo en la costa peruana.

El Formativo Tardío y Final de Casma fue examinado, desde perspectivas distintas pero complementarias, por el siguiente par de ponencias. En la primera, Iván Ghezzi, Alcides Álvarez, Cesar Cornejo, Manuel Lizárraga y Roberto Pimentel expusieron los resultados de varias campañas de investigaciones en Chankillo, el sitio del valle bajo de Casma donde entre 400 y 200 a.C. se construyo uno de los observatorios solares más tempranos de América precolombina (Ghezzi 2007; Ghezzi y Ruggles 2008) $)^{3}$. Estos trabajos proporcionaron nuevos datos sobre el diseño y función de la afamada estructura amurallada del sitio, un edificio fortificado y ceremonial que, gracias a los resultados de diversos estudios realizados desde el siglo XX, se ha convertido en una de las expresiones más remarcables del poder y prestigio de las élites militaristas regionales de la parte final del periodo Formativo. Una de las características del edificio, registradas por las últimas excavaciones, fue el diseño de los accesos al edificio amurallado, algunos de los cuales fueron dispuestos junto a declives abruptos del terreno. Estas evidencias se suman a otras previamente documentadas en el sitio, como las efigies cerámicas de personajes armados registradas en los años 2002 y 2003. Los datos arquitectónicos y cerámicos de Chankillo ponen en relieve el poder y autoridad de algunos grupos sociales locales en capacidad de representar el ethos guerrero de un asentamiento contemporáneo con los sitios del valle de Nepeña previamente mencionados (Fig. 8).

La documentación del diseño arquitectónico y estado de conservación del edificio fortificado de Chankillo fue presentada por Sergio Anchi, Iván Ccachura, Cynthia Navarro, Audry Pallete y Slawomir Swieciochowski, quienes mostraron los resultados de su registro de la condición actual del monumento. Esa labor fue realizada mediante el escaneo tridimensional de la arquitectura. Anchi y sus colegas resaltaron la incidencia de factores como la actividad humana pasada y reciente, el intempe-

3. El registro de arquitectura ceremonial del Arcaico Tardío en Buenavista (valle de Chillón) con recintos, nichos y relieves de barro alineados con posiciones solsticiales demuestra el temprano establecimiento de observaciones astronómicas en la costa peruana (Benfer 2012). 


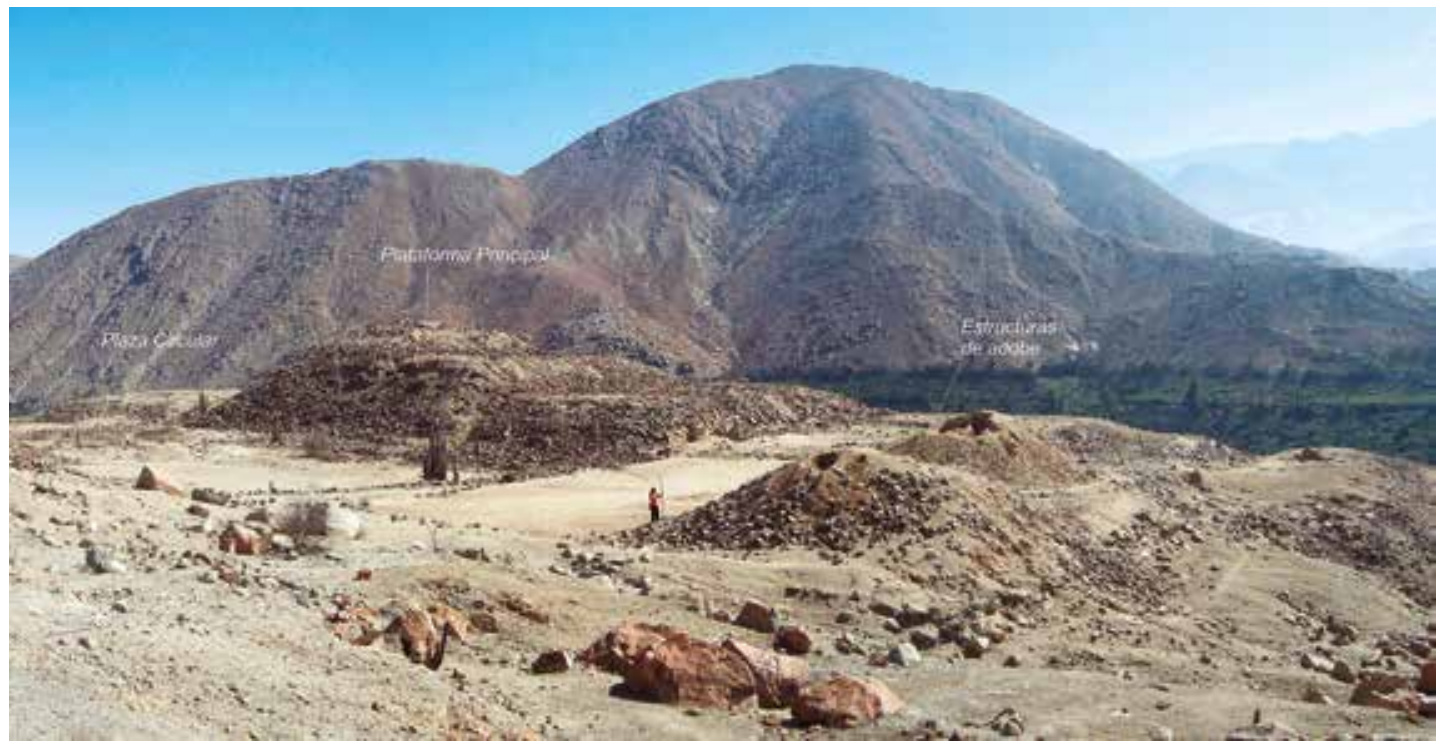

Figura 6. Centro ceremonial Formativo de Pallka. Fotografía por J. Gamboa, 2011.

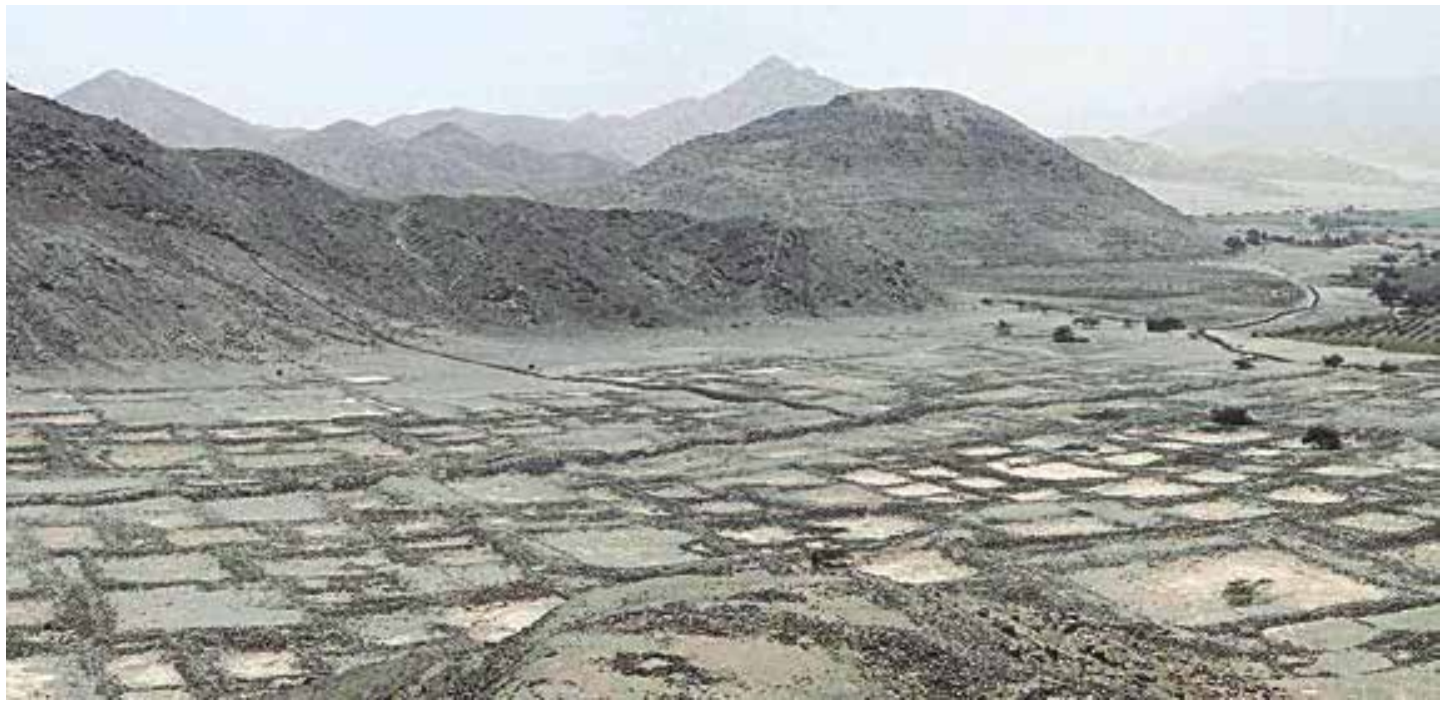

Figura 7. Caylán, valle bajo de Nepeña. Fotografía por J. Gamboa, 2009 


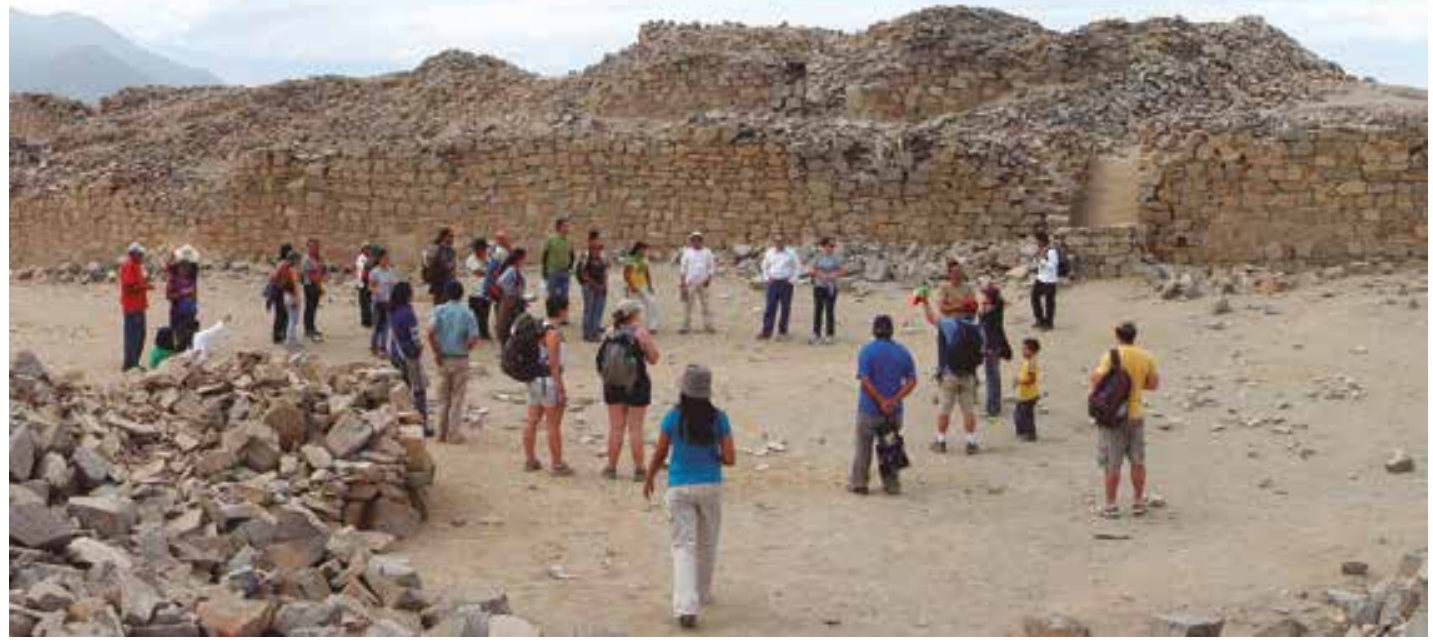

Figura 8. Edificio fortificado de Chankillo. Fotografía por J. Gamboa, 2011.

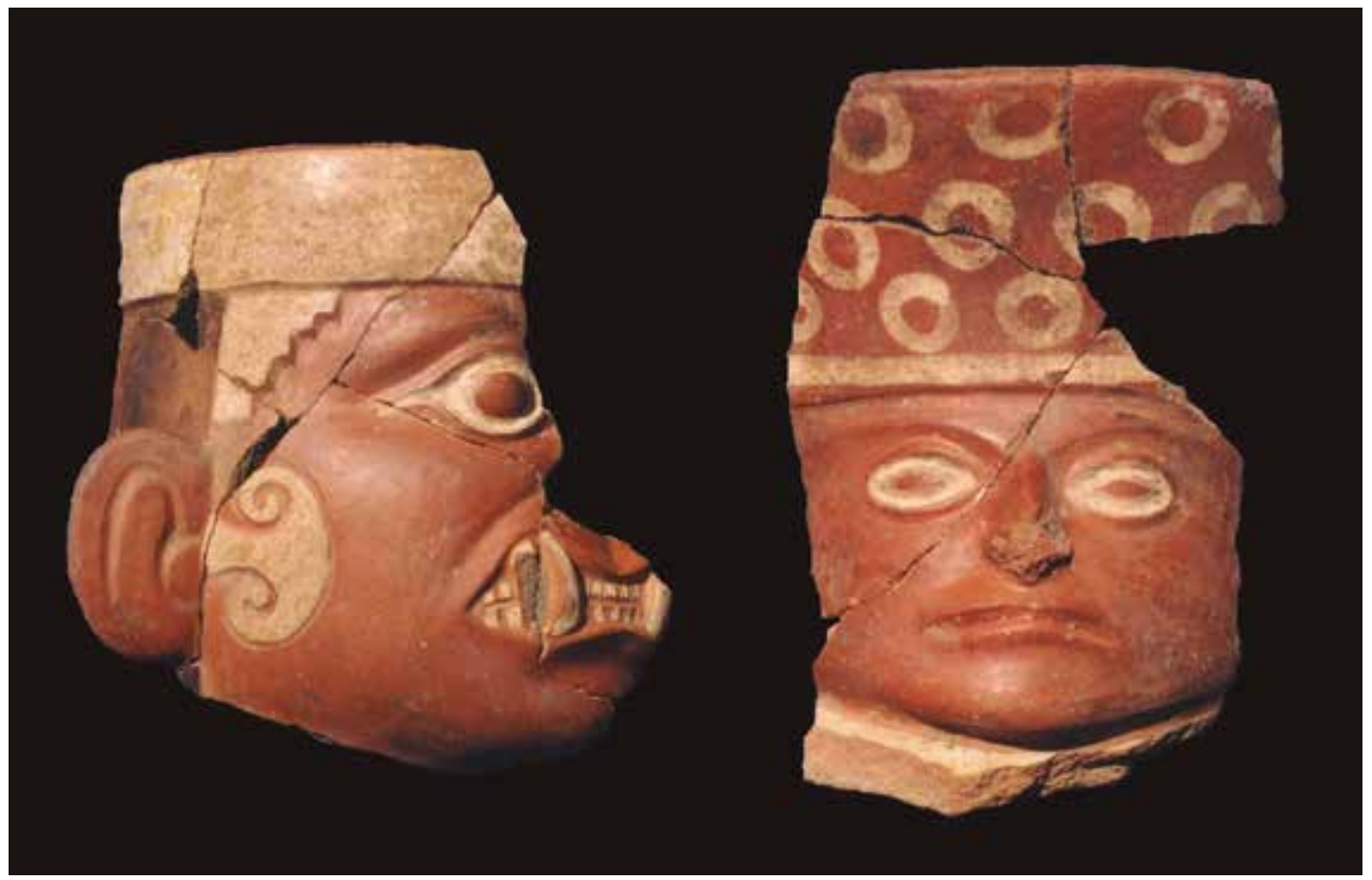

Figura 9. Cerámica Moche III de Castillo de Santa. Proyecto Santa de la Universidad de Montreal. Fotografía por J. Gamboa, 2008. 
rismo y cambios medioambientales, y el crecimiento de líquenes en las rocas como elementos a ser tomados en cuenta en la planificación e implementación de posteriores trabajos de conservación en el sitio. Tras esta contribución, las siguientes ponencias evaluaron la organización de la ocupación Moche en el valle costeño de Santa y el patrón de asentamiento y territorialidad durante el Horizonte Medio en el sector alto del valle de Nepeña y zonas interandinas cercanas.

El tiempo de presencia Moche en la costa de Ancash significó para el área una estrecha interacción con el resto de la costa norte peruana. De hecho, los valles de Santa, Lacramarca, Nepeña y Culebras pasaron a conformar un sector equivalente a la cuarta parte del territorio Moche. Jorge Gamboa, Claude Chapdelaine y Víctor Pimentel presentaron los resultados de una década de investigaciones del Proyecto Santa de la Universidad de Montreal (PSUM) y señalaron las contribuciones de ese programa al estudio de la cronología e identidades étnicas y políticas en la costa nor-central durante los siglos IV a VIII d.C. Parte de la ponencia fue dedicada a exponer las características del sitio Castillo de Santa ${ }^{4}$. Las excavaciones del PSUM en El Castillo de Santa permitieron reconocer que ese asentamiento fue ocupado inicialmente por entidades afiliadas a un estilo local del Formativo Tardío y a la tradición Virú (o Gallinazo Negativo), un periodo que hacia 350 d.C. fue seguido por la introducción masiva de elementos culturales estrechamente relacionados al estilo Moche III (Chapdelaine et al. 2009; Chapdelaine y Pimentel 2003) (Fig. 9). En base a las evidencias de Castillo de Santa y otros asentamientos de Ancash y La Libertad, los autores propusieron la ausencia entre 350 y 550 d.C. de una entidad hegemónica en el área entre los valles de Chicama y Culebras, una situación que no fue obstáculo para que las entidades locales compartieran diversas expresiones ideológicas y de conducta ceremonial. En el valle bajo de Santa esa situación desembocaría hacia 600 d.C. en una reorientación de las relaciones con el valle de Moche, cambio que originaría un nuevo panorama sociopolítico marcado por el abandono de El Castillo de Santa y la emergencia de Guadalupito como nuevo centro ceremonial y administrativo (Chapdelaine 2010; Gamboa 2008).

El periodo entre 650 a 900 dC en Ancash estuvo caracterizado por la interacción entre las poblaciones costeñas y altoandinas locales y la sociedad Wari de la sierra central peruana, un proceso que produjo cambios profundos en identidades, expresiones de autoridad y redes de intercambio. A partir de los datos de la prospección de la cuenca alta del río Nepeña, Alexander Herrera (2006) expuso la importancia de definir y analizar los conceptos de ancestralidad y territorialidad prehispánicos a través del estudio de patrones de asentamiento y relación entre ideologías locales y entorno geopolítico. Su caso de estudio fue el sitio Cerro Limónjirka, en el sector Chakwascucho, un asentamiento que fue examinado en el marco de la organización de las ocupaciones del valle alto de Nepeña y la sección colindante del Callejón de Huaylas entre 700 y 1000 d.C. En Cerro Limónjirka se registró un rasgo formado por un cántaro con la representación moldeada de la cópula de una pareja mítica, un motivo recurrente en la cerámica ceremonial de las poblaciones de la costa y la chaupiyunga nor-central del denominado Horizonte Medio (Carrión 1955). Ese tipo de artefactos habría sido empleado por diversas poblaciones costeras y serranas de Nepeña en la negociación ritual y política de un paisaje compuesto no solo por espacios geográficos y recursos ecológicos sino también por lugares ancestrales y sagrados. En la perspectiva de Herrera, la iconografía de la ofrenda demostraría la relación entre las expresiones de culto al agua y la fertilidad y el establecimiento de formas de legitimidad social por diversas comunidades proclamando vínculos con antepasados locales y foráneos.

4. El Proyecto Santa de la Universidad de Montreal fue iniciado el año 2000 bajo la conducción de Claude Chapdelaine y Víctor Pimentel Spissu. A lo largo de su desarrollo ha incluido trabajos de campo en los sitios San Juanito, Castillo de Santa, Guadalupito, San José de Lacramarca, GUAD-121 y Huaca China, entre otros. 
La jornada fue cerrada por dos presentaciones que examinaron la historia y carácter actual de la actividad arqueológica en la región. Lorenzo Samaniego presentó una memoria del transcurso de las investigaciones en Cerro Sechín desde la llegada de Julio C. Tello hasta los trabajos organizados durante las décadas de 1970 y 1980, periodos en los que se contó con el financiamiento del estado peruano (CRYRZA, Comisión de Rehabilitación y Reconstrucción de la Zona Afectada por el Sismo del 31 de Mayo de 1970) y la Fundación Volkswagen (Cárdenas 1995; Samaniego 1973, 1995) y la colaboración de la Pontificia Universidad Católica del Perú. A lo largo de su ponencia, Samaniego remarcó las condiciones originales y resultados de los primeros trabajos en Cerro Sechín -entre ellos la fundación de su museo de sitio en 1984- y resaltó su influencia en las posteriores actividades de investigación y difusión del patrimonio arqueológico de la costa de Ancash. Ese último punto fue evaluado por Gabriela Freyre, Amanda Gutiérrez y Wendy Augustín, quienes abordaron el rol adquirido por Chankillo en la colectividad casmeña y presentaron sus experiencias en la articulación entre el conocimiento del pasado prehispánico y el desarrollo de la identidad en la población de Casma del siglo XXI.

\section{COMENTARIOS FINALES}

Llevado a cabo en un área caracterizada por un número apreciable de proyectos de investigación y una cantidad mínima de oficinas e instituciones culturales a cargo de la defensa del patrimonio arqueológico, el simposio del 2011 en Casma cumplió dos metas principales: i) crear un espacio de interacción entre los arqueólogos y la comunidad, y ii) contribuir a la difusión del conocimiento del pasado entre la audiencia local ${ }^{5}$. Ambos objetivos fueron alcanzados, produciendo -durante un lapso de tiempo- el tan necesitado intercambio de opiniones y perspectivas entre el "público" y los "especialistas". En los años siguientes, la Casa de la Cultura de Casma ha proseguido con sus actividades de difusión de la investigación arqueológica, aunque para ello ha debido afrontar continuas limitaciones presupuestales y logísticas. A pesar de ello, esa institución ha logrado convocar en varias temporadas a investigadores no solo de los sitios prehispánicos locales sino también a aquellos dedicados a los valles vecinos de Nepeña y Huarmey. En todas esas ocasiones se contó con el apoyo del museo de Cerro Sechín, principalmente a través de la prestación gratuita de las instalaciones necesarias para el cumplimiento de las reuniones. A pesar de los esfuerzos desplegados, dos de las metas inicialmente planteadas por estos gestores culturales locales no han sido alcanzadas: lograr que la historia prehispánica de la costa de Ancash sea incluida más ampliamente en los discursos oficiales de la educación y evitar la continuidad del deterioro y destrucción de varios componentes del patrimonio arqueológico regional.

Recordar que la gestión del simposio de arqueología en Casma partió de la iniciativa de un grupo comunitario nos conduce a reflexionar sobre la realidad de los vínculos y diferencias entre los grupos formados por investigadores, promotores y gestores culturales. El encuentro analizado expuso, de manera explícita o implícita, parte de las demandas locales de aproximación al tema patrimonial a partir de propuestas ciudadanas; al mismo tiempo, el simposio puso en evidencia los retos de la articulación de grupos con agendas distintas de acción y discurso, cuyas metas varían entre la investigación académica, los planes educativos, el desarrollo económico local, y la (re)creación de identidades en base al pasado regional.

5. El Ministerio de Cultura cuenta en la costa de Ancash con dos sedes administrativas (Chimbote y Cerro Sechín en Casma), un museo de sitio (Cerro Sechín) y dos estaciones de control de sitios arqueológicos (Pañamarca y Paramonga). La costa de Ancash comprende los valles de Santa (margen sur del valle bajo y medio), Lacramarca, Nepeña, Casma, Culebras, Huarmey y Fortaleza y el sistema de planicies y quebradas en la intercuenca Huarmey-Fortaleza. El número total del personal asignado por el estado para supervisar ese territorio ha fluctuado entre dos y cuatro arqueólogos durante los años 2014 a 2016. 
Sin embargo, el balance del los resultados y propósito del evento fue positivo. En ese aspecto, la finalidad del encuentro se enmarcó en una tendencia global cada vez más fuerte. A pesar de las diferencias señaladas y las dificultades inherentes a alcanzar una definición consensuada de comunidad, existe un amplio acuerdo en la necesidad de fomentar la participación activa de las poblaciones locales en la elaboración y puesta en marcha de planes y programas culturales (Agbe-Davis 2010; Castillo 2004; Marshall 2002; Meskell 2010; Silverman y Ruggles 2007). La discusión de las políticas y derechos culturales ha sido conducida a través de ese cambio de paradigma hacia el análisis y valoración de las experiencias y estrategias locales en la identificación, conservación y uso del patrimonio tangible e intangible. Como resultado de ese debate ha sido reconocido que la confluencia de las aproximaciones desde abajo y desde arriba, aunque provista de sus propias dificultades, es indispensable para diseñar e implementar políticas culturales efectivas (Gamboa 2015:10-11, 75-85; Lane 2013; Ortega 2003). Volviendo a nuestro caso de estudio y a un lustro de su origen, la gestión local examinada revela la necesidad de dar continuidad a prácticas sociales -esporádicas y aún eventuales- ahora reconocidas como formas válidas de acercamiento de la sociedad a la materialidad y significado del pasado.

Como se señaló al inicio del artículo, tanto el público joven como adulto del simposio de Casma demostró interés en discutir una historia poco incluida en el sistema educativo nacional. En varios casos, el reconocimiento de la importancia de esa historia por los actores sociales involucrados apareció basado en la proximidad entre la residencia o área de trabajo de los participantes y los sitios y paisajes arqueológicos discutidos, un tipo de relación que suele contribuir a la creación de varios niveles de sentido de pertenencia a un espacio (Low y Lawrence-Zuñiga 2003). A un nivel general, esa actitud también refleja expresiones ampliamente compartidas de apreciación de algunas evidencias arqueológicas como componentes de las identidades regionales y nacionales. Sin embargo, los diálogos y posicionamientos sobre espacios en torno a los cuales se evocan y construyen identidades no deben ser considerados unidireccionales. Es evidente que los arqueólogos manejan sus propias formas de sentido de lugar en torno a los sitios que investigan. Para los especialistas en arqueología el reconocimiento de las percepciones de otras personas en los temas de arqueología y patrimonio puede convertirse en un campo de aprendizaje mutuo y ampliación de la labor de difusión del conocimiento más allá de la esfera académica y las ciudades principales. En conjunto, las experiencias y perspectivas señaladas hacen referencia a su vez un trasfondo aún poco discutido en Perú: la puesta en marcha de estrategias representativas o, alternativamente, participativas del público en las políticas de manejo del patrimonio arqueológico nacional.

La costa y las estribaciones serranas de la costa de Ancash presentan un valioso y amplio legado arqueológico que comprende evidencias materiales de las primeras ocupaciones humanas diez milenios atrás hasta el pasado reciente. El impacto social de los estudios arqueológicos conducidos en el área puede ser medido en diversos niveles -que abarcan desde la creación temporal de puestos de trabajo hasta la exposición de materiales procedentes de excavaciones y prospecciones. Por otro lado, estas formas de interacción han ocurrido, hasta ahora, en un número reducido de localidades. Aunque la mayoría de casos han dado origen a diversos tipos de asociación entre arqueólogos y comunidades locales, es evidente que ese acercamiento ha sido limitado, comprendiendo con frecuencia audiencias restringidas a algunos segmentos de las comunidades rurales o urbanas más cercanas a los sitios arqueológicos estudiados.

La continuidad de las investigaciones y su presentación pública podrían ser en los próximos años algunos de los medios que permitan implementar una política efectiva de protección de los sitios arqueológicos desde y por las poblaciones locales. Ese cambio de actitud será, finalmente, lo único que podrá conducir a que el patrimonio arqueológico de Ancash sea reconocido por el público como 
un bien social compartido y no renovable y un elemento activo en el reconocimiento de la memoria histórica y el desarrollo sostenible de las comunidades involucradas.

\section{AgRAdECIMIENTOS}

La redacción de este trabajo no habría sido posible sin la cordial colaboración del Profesor Lorenzo Castillo Cordero, Víctor López Velásquez y los demás integrantes de la Casa de la Cultura de Casma. Asimismo, se reconoce el apoyo proporcionado por Luis Burgos, Director del Museo de Cerro Sechín, y los colegas participantes en el simposio. Irene Elena Brichetti, Jason Nesbitt, David Pacifico, Thomas Pozorski, Sarahh Scher, Henry Tantaleán y Melissa Vogel contribuyeron a la elaboración de este manuscrito a través de valiosos comentarios y sugerencias. Cualquier error u omisión es de responsabilidad exclusiva del autor.

\section{BibliografíA}

\section{AGBE-DAVIES, Anna}

2010 "Concepts of community in the pursuit of an inclusive archaeology". International Journal of Heritage Studies 16(6):373-389.

\section{ALVA, Walter y Christopher DONNAN}

1993 Royal Tombs of Sipán. Fowler Museum of Cultural History. Los Angeles: University of California, Los Angeles.

BENFER, Robert

2012 "Monumental architecture arising from and early astronomical-religious complex in Peru, 2200-1750 BC". En: R. Rosenswig y R. Burger (eds.) Early New World Monumentality. Gainesville: University of Florida Press.

BRIA, Rebecca

2013 "Proyecto de Investigación Arqueológica Regional Ancash". http://www.piaraperu.org (accedido 15 febrero 2016].

\section{CÁRDENAS, Mercedes}

1995 “Iconografía lítica de Cerro Sechín: Vida y muerte”. En: S. Lerner, M. Cárdenas y P. Kaulicke, eds. Arqueología del Cerro Sechín. Tomo II, pp. 45-124. Lima: Fondo Editorial de la Pontificia Universidad Católica del Perú y Fundación Volkswagenwerk.

\section{CARRIÓN CACHOT, Rebeca}

1955 "El culto al agua en el Antiguo Perú". Revista del Museo Nacional de Antropología y Arqueología $2(2): 50-140$.

CASTILLO, Luis Jaime

2004 “Arqueología y desarrollo comunitario sostenible en San José de Moro”. En: L.J. Castillo (ed.) Programa Arqueológico San José de Moro. Temporada 2004, pp. 168-193 (http://sanjosedemoro.pucp.edu.pe/descargas/reportes/INFORME2004.pdf (accedido 28 diciembre 2015).

CHAPDELAINE, Claude

2010 "Moche political organization in the Santa Valley. A case of direct rule through gradual control of the local population". En: J. Quilter y L.J. Castillo (eds.) New Perspectives on Moche Sociopolitical Organization, pp. 252-279. Washington DC: Dumbarton Oaks. 
CHAPDELAINE, Claude y Víctor PIMENTEL

2003 “Un tejido único Moche III del sitio Castillo de Santa". Bulletin de l'Institut Français d'Études Andines 32(1):23-50.

CHAPDELAINE, Claude, Víctor PIMENTEL y Jorge GAMBOA

2009 "Gallinazo cultural identity in the Lower Santa Valley: Ceramics, architecture, burial patterns, and sociopolitical organization". J.F. Millaire y M. Morlion (eds.) Gallinazo. An Early Cultural Tradition on the Peruvian North Coast, pp. 181-206. Cotsen Institute of Archaeology Press. University of California, Los Angeles.

CHÁVEZ, Jack

2011 "Pallka, un sitio del periodo Formativo en la parte media alta del valle de Casma". En: I. Ghezzi y M. Giersz (eds.) Andes 8. Arqueología de la costa de Ancash, pp. 97-112. Lima: Centro de Estudios Precolombinos de la Universidad de Varsovia e Instituto Francés de Estudios Andinos.

CHICOINE, David y Hugo IKEHARA

2010 "Nuevas evidencias sobre el Periodo Formativo del valle de Nepeña: Resultados preliminares de la primera temporada de investigaciones en Caylán”. Boletín de Arqueología PUCP 12:349-369.

FUCHS, Peter, Rebata PATZSCHKE, Germán YENQUE y Jesús BRICEÑO

2010 “Del Arcaico Tardío al Formativo Temprano: Investigaciones en Sechín Bajo, valle de Casma”. Boletín de Arqueología PUCP 13: 55-86.

GAMBOA, Jorge

2008 "Plazas y cercaduras. Una aproximación a la arquitectura pública Moche IV y V en los valles de Moche y Santa". En: L.J. Castillo, H. Bernier, G. Lockard y J. Rucabado (eds.) Arqueología Mochica. Nuevos Enfoques, pp. 203-217. Lima: Fondo Editorial de la Pontificia Universidad Católica del Perú.

2015 Archaeological Heritage in a Modern Urban Landscape. The Ancient Moche in Trujillo, Peru. New York: Springer-SpringerBriefs in Archaeology. Archaeological Heritage Management Series.

GHEZZI, Iván

2007 “La naturaleza de la guerra prehispánica temprana: La perspectiva desde Chankillo”. Revista Andina 44:199-255.

GHEZZI, Iván y Clive RUGGLES

2008 “Chankillo: A 2300 year-old solar observatory in coastal Peru”. Science 315:120-124.

HERRERA, Alexander

2006 “Territorio e identidad: Apuntes para un modelo de la complejidad social andina". A. Herrera, C. Orsini y K. Lane (eds.) Complejidad social en la arqueología y antropología de la sierra de Ancash, pp. 3-18. Milán: Castello Sforzesco, Raccolte Extraeuropee y Centro Punku, 
IKEHARA, Hugo

2010 “Kushipampa: El final del Periodo Formativo en el valle de Nepeña”. Boletín de Arqueología PUCP 12:371-404.

LANE, Kevin

2013 "Entre el agua y la pared: Patrimonio, desarrollo, campesinos y arqueólogos en la Cordillera Negra, Perú”. En: A. Herrera (ed.) Arqueología y desarrollo en América del Sur. De la práctica a la teoría, pp. 97-117. Lima: Universidad de los Andes e Instituto de Estudios Peruanos.

LAU, George

2010 "House forms and Recuay culture: Residential compounds at Yayno (Ancash, Peru), a fortified hilltop town, AD 400-800". Journal of Anthropological Archaeology 29:327-351.

LOW, Setha y Denise LAWRENCE-ZÚÑIGA (eds.)

2003 The Anthropology of Space and Place: Locating Culture. Malden: Blackwell Publishing.

MAMANI, Carlos

1996 "History and prehistory in Bolivia. What about the Indians?" R. Preucel y I. Hodder (eds.) Contemporary Archaeology in Theory. A Reader, pp. 632-645. Oxford: Blackwell.

MARCUS, Joyce

2015 "Studying the individual in prehistory: A tale of three women from Cerro Azul, Peru". Nawpa Pacha 35(1):1-22.

MARSHALL, Yvonne

2002 “What is community archaeology?” World Archaeology 34(2):211-219.

MESKELL, Lynn

2010 “Human rights and heritage ethics”. Anthropological Quarterly 83:839-860.

MONTENEGRO, Mónica y María Clara RIVOLTA

2013 "Patrimonio arqueológico y desarrollo: Pasados que se hacen presente. Experiencias desde el noroeste argentino”. En: A. Herrera (ed.) Arqueología y desarrollo en América del Sur. De la práctica a la teoría, pp. 17-34. Lima: Universidad de los Andes e Instituto de Estudios Peruanos.

NELSON, Andrew, Chris NELSON, Luis Jaime CASTILLO y Carol MACKEY

2000 "Osteobiografía de una hilandera prehispánica. Iconos". Revista Peruana de Conservación y Arqueología 4:30-43.

ONUKI, Yoshio

2006 “The Kuntur Wasi Museum in northern Peru”. En: H. Silverman (ed.) Archaeological Site Museums in Latin America, pp. 64-71. Gainesville: University of Texas Press.

ORTEGA, Verónica

2003 “El patrimonio arqueológico de Teotihuacán. Responsabilidad Social”. Arqueología Mexicana 9(64):58-61. 
PACIFICO, David

2008 Archaeology and the Public's Interests at El Purgatorio, Casma, Peru. Tesis de Maestria. Chicago: University of Chicago, Department of Anthropology.

POZORSKI, Shelia y thomas Pozorski

1987 Early Settlement and Subsistence in the Casma Valley, Perú. Iowa City: University of Iowa Press.

RICK, John, Christian MESIA, Daniel CONTRERAS, Silvia KEMBEL, Rosa MENDOZA, Matthew SAYRE y John WOLF

2009 “La cronología de Chavín de Huántar y sus implicancias para el Periodo Formativo". Boletín de Arqueología PUCP 13:87-132.

SAMANIEGO, Lorenzo

1973 Los nuevos trabajos arqueológicos en Sechín, Casma, Perú. Trujillo: Larsen.

1995 “Escultura del edificio central de Cerro Sechín”. En: S. Lerner, M. Cárdenas y P. Kaulicke (eds.) Arqueología del Cerro Sechín. Tomo II, pp. 19-41. Lima: Fondo Editorial de la Pontificia Universidad Católica del Perú y Fundación Volkswagenwerk.

SILVERMAN, Helaine y D. FAIRCHILD RUGGLES (eds.)

2007 Cultural Heritage and Human Rights. New York: Springer.

SZPAT, Paul, David CHICOINE, Jean-François MILLAIRE, Christine WHITE, Rebecca PARRY y Fred LONGSTAFFE

2015 "Early Horizon camelid management practices in the Nepeña Valley, north-central coast of Peru”. Enviromental Archaeology 20(1):13-29.

SUÁREZ, Mónica

2008 “Investigaciones arqueológicas en Pallka, valle medio de Casma, Ancash". Supay. Revista de Humanidades y Ciencias del Hombre 6:25-37. Lima: Universidad Nacional Federico Villarreal.

TELLO, Julio C

1956 “Arqueología del valle de Casma. Culturas: Chavín, Santa o Huaylas Yunga, y Sub-Chimú. Informe de los trabajos de la Expedición Arqueológica al Marañón de 1937”. Publicación Antropológica del Archivo Tello. Lima: Universidad Nacional Mayor de San Marcos.

UCEDA, Santiago

1992 “Industrias líticas de Casma”. En: D. Bonavia (ed.) Estudios de Arqueología Peruana, pp. 45-67. Lima: FOMCIENCIAS.

VOGEL, Melissa y David PACIFICO

2004 "From theory to practice: Public interest archaeology in Peru". Anthropology in Action 11(2):4453.

WESTER, Carlos

2010 Chotuna-Chornancap: Templos, rituales y ancestros Lambayeque. Lima: Unidad Ejecutora Naylamp y Museo Brüning de Lambayeque. 
